Nat. Hazards Earth Syst. Sci., 18, 1187-1199, 2018

https://doi.org/10.5194/nhess-18-1187-2018

(C) Author(s) 2018. This work is distributed under

the Creative Commons Attribution 4.0 License.
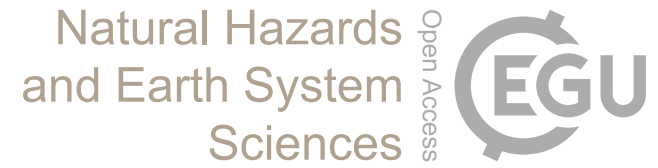

\title{
State fusion entropy for continuous and site-specific analysis of landslide stability changing regularities
}

\author{
Yong Liu, Zhimeng Qin, Baodan Hu, and Shuai Feng \\ School of Mechanical Engineering and Electronic Information, China University of Geosciences, Wuhan, 430074, China
}

Correspondence: Zhimeng Qin (imqinzhimeng@163.com)

Received: 6 October 2017 - Discussion started: 1 November 2017

Revised: 21 March 2018 - Accepted: 23 March 2018 - Published: 19 April 2018

\begin{abstract}
Stability analysis is of great significance to landslide hazard prevention, especially the dynamic stability. However, many existing stability analysis methods are difficult to analyse the continuous landslide stability and its changing regularities in a uniform criterion due to the unique landslide geological conditions. Based on the relationship between displacement monitoring data, deformation states and landslide stability, a state fusion entropy method is herein proposed to derive landslide instability through a comprehensive multi-attribute entropy analysis of deformation states, which are defined by a proposed joint clustering method combining $K$-means and a cloud model. Taking Xintan landslide as the detailed case study, cumulative state fusion entropy presents an obvious increasing trend after the landslide entered accelerative deformation stage and historical maxima match highly with landslide macroscopic deformation behaviours in key time nodes. Reasonable results are also obtained in its application to several other landslides in the Three Gorges Reservoir in China. Combined with field survey, state fusion entropy may serve for assessing landslide stability and judging landslide evolutionary stages.
\end{abstract}

\section{Introduction}

Landslides are some of the greatest natural hazards, accounting for massive damages of properties every year (Dai et al., 2002). Analysis of landslide stability as well as its changing regularities plays a significant role in risk assessment at site-specific landslides (Wang et al., 2014). For this concern, many stability analysis methods have been proposed, such as Saito's method, limit equilibrium method (LEM) and finite element method (FEM) (Saito, 1965; Duncan, 1996; Grif- fiths and Fenton, 2004). Saito's method is an empirical forecast model and is suitable for the prediction of sliding tendency and then the failure time. Based on homogeneous soil creep theory and displacement curve, it divides displacement creep curves into three stages - deceleration creep, stable creep and accelerating creep - and establishes a differential equation for accelerating creep. The physical basis of Saito's method helped it to successfully forecast a landslide that occurred in Japan in December 1960, but also makes it strongly dependent on field observations. LEM is a kind of calculation method to evaluate landslide stability based on mechanical balance principle. By assuming a potential sliding surface and slicing the sliding body on the potential sliding surface firstly, LEM calculates the shear resistance and the shear force of each slice along the potential sliding surface and defines their ratio as the safety factor to describe landslide stability. LEM is simple and can directly analyse landslide stability under limit condition without geotechnical constitutive analysis. However, this neglect of geotechnical constitutive characteristic also restricts it to a static mechanics evaluation model that is incapable of evaluating the changing regularities of landslide stability. LEM involves too many physical parameters, such as cohesive strength and friction angle, which makes it greatly limited in landslide forecast. As a typical numerical simulation method, FEM subdivides a large problem into smaller, simpler parts that are called finite elements. The simple equations that model these finite elements are then assembled into a larger system of equations that models the entire problem. FEM then uses variational methods from the calculus of variations to approximate a solution by minimising an associated error function. In landslide stability analysis, FEM not only satisfies the static equilibrium condition and the geotechnical constitutive characteris- 
tic but also adapts to the discontinuity and heterogeneity of the rock mass. However, FEM is quite sensitive to various involved parameters and the computation will increase greatly to get more accurate results. If parameters and boundaries are precisely determined, LEM and FEM can provide results with high reliability. Other stability analysis methods such as the strength reduction method also have been rapidly applied (Dawson et al., 2015). These methods provide the theoretical basis for analysing landslide stability and have been widely applied in engineering geology (Knappett, 2008; MoralesEsteban et al., 2015).

Despite of the great contributions made by these stability analysis methods, there are a few matters cannot be neglected. Firstly, the safety factor is the most adopted index to indicate landslide stability (Hsu and Chien, 2016), but it mainly indicates safe (larger than 1) or unsafe (smaller than 1) and is incapable of showing the degree of stability or instability (Li et al., 2009; Singh et al., 2012). Secondly, external factors such as rainfall (Priest et al., 2011; Bernardie et al., 2015; Liu et al., 2016) and fluctuation of water level (Ashland et al., 2006; Huang et al., 2017b) will also change landslide stability. However, currently only a few studies have mentioned real-time landslide stability (Montrasio et al., 2011; Chen et al., 2014). Thirdly, methods like LEM and FEM involve too many physical parameters whose uncertainties make these methods hard to match with the real-time conditions of landslide. It becomes of great interest to find a new method to evaluate landslide stability that only requires a few parameters, can easily be matched with landslide real-time conditions and can indicate the extent as well as the changing regularities of landslide stability.

Displacement is the most direct and continuous manifestation of landslide deformation promoted by external factors and has been widely used in landslide analysis (Asch et al., 2009; Manconi and Giordan, 2015; Huang et al., 2017a). Due to its easy acquisition, quantification and high reliability, displacement monitoring data have become some of the most recognised evidence for landslide stability analysis and early warning. Macciotta et al. (2016) suggested that velocity threshold be used as a criterion for an early warning system and that the annual horizontal displacement threshold for Ripley landslide (GPS 1) can be $90 \mathrm{~mm}$ and that between May and September can be $25 \mathrm{~mm}$. Based on the analysis of a large number of displacement monitoring data, $\mathrm{Xu}$ and Zeng (2009) proposed that deformation acceleration be used as an indicator of landslide warning; the acceleration threshold of Jimingsi landslide was regarded as $0.45 \mathrm{mmd}^{-2}$ and that of another landslide in Daye iron mine as $0.2 \mathrm{mmd}^{-2}$. Federico et al. (2012) presented a systematic introduction to the prediction of landslide failure time according to the displacement data. However, although displacement data have been widely used in landslide analysis, it is hard to define a unified displacement threshold due to the unique geological conditions, and many studies draw their conclusions directly based on original data and personal engineering geological experience.

Entropy has been widely used to describe the disorder, imbalance and uncertainty of a system (Montesarchio et al., 2011; Ridolfi et al., 2011). Previous works have introduced entropy into landslide susceptibility mapping to evaluate the weights of indexes (Pourghasemi et al., 2012; Devkota et al., 2013). From the viewpoint of system theory, a landslide can be regarded as an open system that exchanges energy and information with external factors. Shi and Jin (2009) proposed a generalized information entropy approach (GIE) to evaluate the "energy" of multi-triggers of landslide and found that the GIE index showed a mutation before landslide failure in a case study. But this GIE method is aimed at landslide triggering factors and thus cannot directly indicate landslide stability.

In this paper, a state fusion entropy (SFE) approach is proposed for continuous and site-specific analysis of landslide stability changing regularities. It firstly defines deformation states as an integrated numerical feature of landslide deformation. Considering the multiple attributes of deformation states, entropy is adopted for landslide stability (instability) analysis. Correspondingly, a historical maximum index is introduced to identify key time nodes of stability changes.

\section{Methods}

In this paper, a landslide is regarded as an open dynamic system, and landslide stability (instability) is the source of the system. Under the influence of external factors, landslide stability will respond to these triggers by generating deformation states. Eventually, deformation states will be manifested in the form of landslide displacement. Therefore, to analyse landslide stability based on displacement monitoring data, defining deformation states is the primary foundation. In order to adapt to the unique geological conditions of different landslides, a joint clustering method combining $K$-means clustering and cloud modeling is proposed. Aiming for three typical characteristics of deformation states, entropy analysis is then conducted and fused to analyse landslide instability and its changing regularities. A result interpretation method is proposed correspondingly. The flow chart is shown in Fig. 1.

\subsection{Deformation state definition based on $K$-means combined with a cloud model}

Many deformation states exist during the development of landslide (Wu et al., 2016) and link up landslide stability and displacement monitoring data. On the one hand, deformation states indicate temporary landslide stability. On the other hand, deformation states can be manifested by displacement monitoring data. Therefore, the excavation of deformation states can be the primary step for analysing landslide stability 


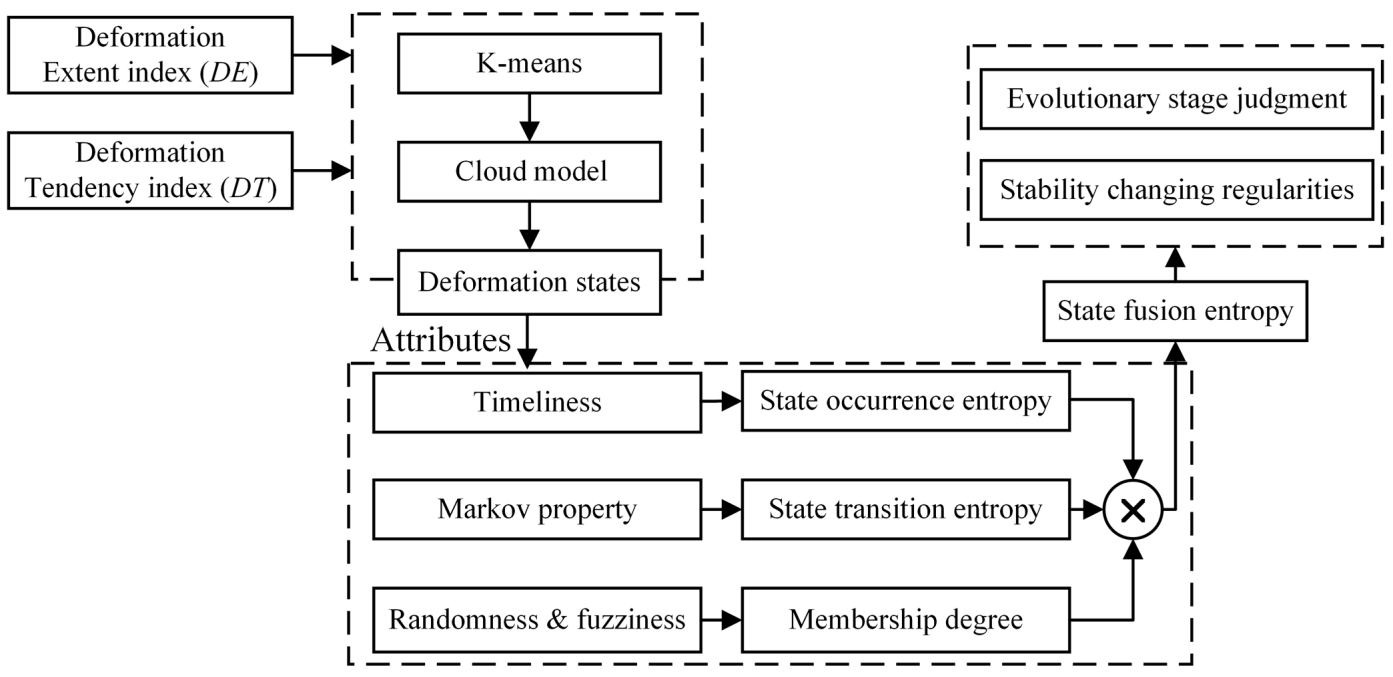

Figure 1. Flow chart of state fusion entropy method.

analysis and its changing regularities according to displacement data. Due to the unique geological conditions of different landslides, a unified definition of deformation states seems infeasible. In view of this, the data-driven $K$-means clustering method and a cloud model are integrated to investigate deformation states.

$K$-means is one kind of unsupervised clustering methods of vector quantisation and is popular in data mining. It aims to partition $N$ observations into $K$ clusters in which each observation belongs to the cluster with the nearest mean (Steinley, 2006; Hartigan and Wong, 2013). Given a set of observations $\left(x_{1}, x_{2}, \ldots, x_{N}\right)$, where each observation is a $d$ dimensional real vector, $K$-means clustering aims to partition the $N$ observations into $K(K \leq N)$ sets $S=\left\{S_{1}, S_{2}, \ldots, S_{K}\right\}$. Formally, the objective is to find the $K$ sets to minimise intraclass distance and maximise inter-class distance through iterations. The objective of $K$-means can be expressed as Eq. (1).

$\underset{s}{\arg \min } D_{\text {intra }}=\underset{s}{\arg \min } \sum_{i=1}^{K} \sum_{x \in S_{i}}\left|x-c_{i}\right|^{2}$,

$\underset{s}{\arg \min } D_{\text {inter }}=\underset{s}{\arg \min } \sum_{i=1}^{K} \sum_{j=i}^{K}\left|c_{i}-c_{j}\right|^{2}$,

where $c_{i}$ is the mean of points in $S_{i}$; $D_{\text {intra }}$ is the pairwise squared deviations of points in the same cluster, representing the consistency of each cluster; and $D_{\text {inter }}$ is the squared deviations between points in different clusters, reflecting the differences among clusters.

$K$-means clustering method is simple, fast and efficient. All observations will be labelled after clustering. However, since the clustering process is unsupervised, the cluster labels of observations are unstable and have a certain randomness. Additionally, the $K$-means algorithm lacks the index to distinguish observations in the same cluster, which leads to high fuzziness of cluster labels. Focussing the randomness

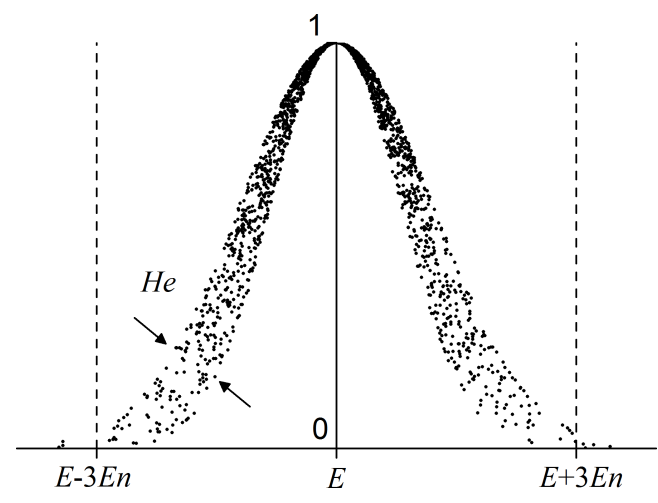

Figure 2. Digital features of one-dimensional cloud.

and fuzziness of cluster labels, a cloud model is introduced to offer help.

The cloud model was proposed in 1995 to analyse the uncertain transformation between qualitative concepts and their quantitative expressions ( $\mathrm{Li}$ et al., 1995). Among all cloud models, the normal cloud model is the most popular due to its universality (Li and Liu, 2004). Let $U$ be a universe of quantitative values, and $C$ be the qualitative concept of $U$. For any element $x$ in $U$, if there exists a random number $y=\mu_{A}(x), y \in[0,1]$ with a stable tendency, then $y$ is defined as the membership (certainty) of $x$ to $C$ and the distribution of $y$ on the universe $U$ is defined as a cloud. The cloud model uses the expectation $(E)$, entropy (En) and hyper-entropy (He) to characterise a qualitative concept and integrates the ambiguity and randomness of the concept. Expectation is the central value of the concept in the universe and is the value that best represents the qualitative concept. Entropy reflects the ambiguity of the qualitative concept and indicates the range of values that the concept accepts in the universe. Hyper-entropy indicates the randomness of mem- 


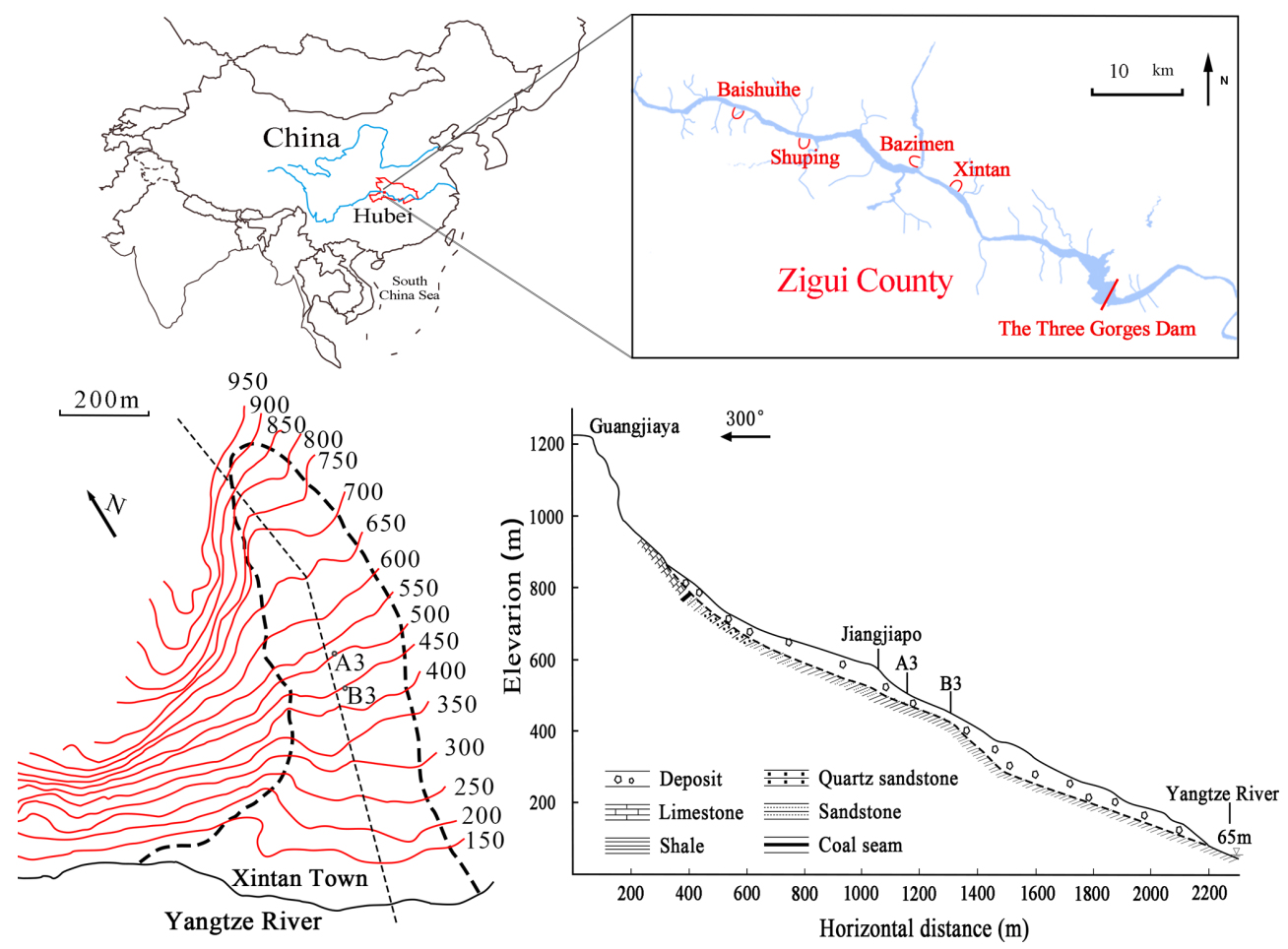

Figure 3. Location and plane/section of Xintan landslide.

bership. The diagram of digital features of one-dimensional cloud is shown in Fig. 2. Given the digital features of a onedimensional normal cloud [Ex, Enx, Hex], cloud droplets can be generated by a forward cloud generator (CG) in the following order: (1) generate a normal random number $x$ with Ex as the mean and Enx as variance; (2) generate a normal random number Enx' with Enx as the mean and Hex as variance; (3) calculate the membership as Eq. (2) and each $(x, y)$ is defined as a cloud droplet; (4) repeat the above steps until required number of cloud droplets are generated. Correspondingly, the process of calculating digital features based on cloud droplets is called the backward cloud generator $\left(\mathrm{CG}^{-1}\right)$.

$y=\exp \left\{-\frac{(x-E x)^{2}}{2 E n x^{\prime 2}}\right\}$

$K$-means can automatically derive labels (concepts) from data but cannot distinguish items with the same label. The cloud model can utilise the distribution characteristics of data and express the membership of each data item to corresponding concept, but cannot work without defining concepts. Therefore, a joint clustering method combining $K$-means and the cloud model is proposed to define landslide deformation states according to displacement monitoring data. To describe clearly this method, two functional data types are defined for landslide displacement data. One is to indicate deformation extent (DE) and the other deformation tendency
(DT). Positive DT indicates an increasing deformation. The process of defining deformation states is as follows.

Step 0. Unite DE and DT at the same time as an item, i.e., (DE, DT).

Step 1. Cluster all items based on $K$-means and obtain cluster labels (K_label) and the distance of each item to corresponding cluster centroid $\left(d_{\text {ic }}\right)$.

Step 2. For each cluster (cloud)

a. select a proportion of items as the typical items based on $d_{\mathrm{ic}}$;

b. conduct $\mathrm{CG}^{-1}$ on typical items to obtain the digital features of this cloud;

c. conduct CG to generate cloud droplets based on the digital features for visual analysis.

Step 3. Calculate and normalise the memberships of each item to all clouds, and define the cloud label with the largest membership as the deformation state of corresponding item.

As can be seen from above procedures, the definition of deformation states is basically driven by displacement monitoring data and thus can adapt the unique geological conditions of different landslides. Membership can be used to distinguish the displacement data with the same deformation 


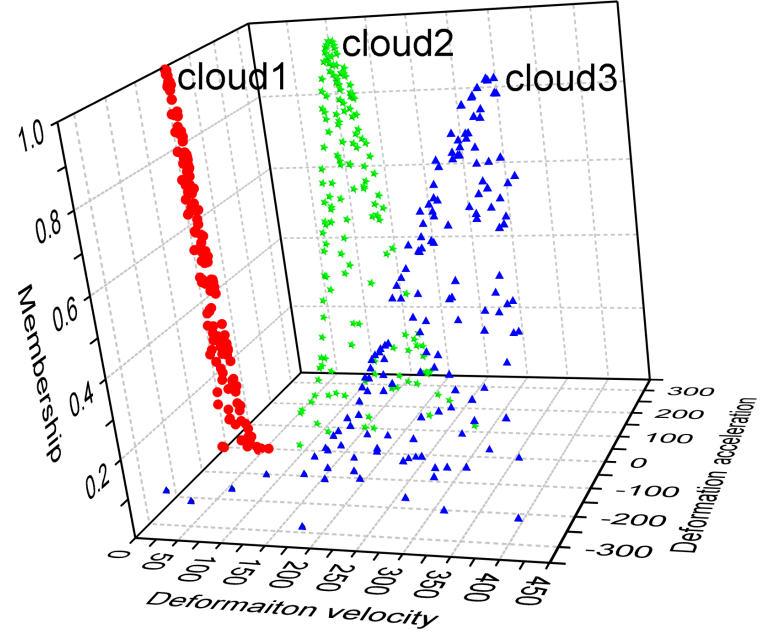

Figure 4. Regenerated clouds of each cluster.

state. Since displacement data are acquired in chronological order, the result is also a time-related state sequence.

As for the deformation state sequence, three typical attributes need to be noticed: the timeliness, the Markov property and fuzziness. Timeliness is the primary attribute of each deformation state and is the basis of stability analysis. The Markov property is caused by the continuity and hysteresis characteristic of external trigging factors such as rainfall and fluctuation of water level (Bordoni et al., 2015). The fuzziness is introduced in the process of defining deformation states.

\subsection{Fusion entropy analysis of deformation state sequence}

Entropy is an indicator of the degree of system chaos. Introduced in the communication system by Shannon in 1948, entropy has become the basis of information theory (Shannon, 1948). Let $X$ be a discrete random variable, where $x$ is one state of $X$ and $p(x)$ is the probability when $X=x$. The information entropy of $X$ can be calculated by Eq. (3).

$I(x)=-\log p(x)$,

$H(X)=\sum_{x \in X} p(x) \cdot I(x)$,

where $I(x)$ is the information amount of $x$ and $H(X)$ is the entropy of $X$. As shown in Eq. (3), the information amount increases with the decrease of probability. $H(X)$ is the statistical average of the information amount of each state, representing the overall uncertainty of $X$. The $p(x)$ weighted $I(x)$ can be regarded as the individuation of state $x$ to overall uncertainty $H(X)$.

As for landslide deformation states, each contains some information about landslide stability. Slight deformation occurs frequently but indicates a relatively stable state of a landslide. Severe deformation occurs rarely but indicates a really

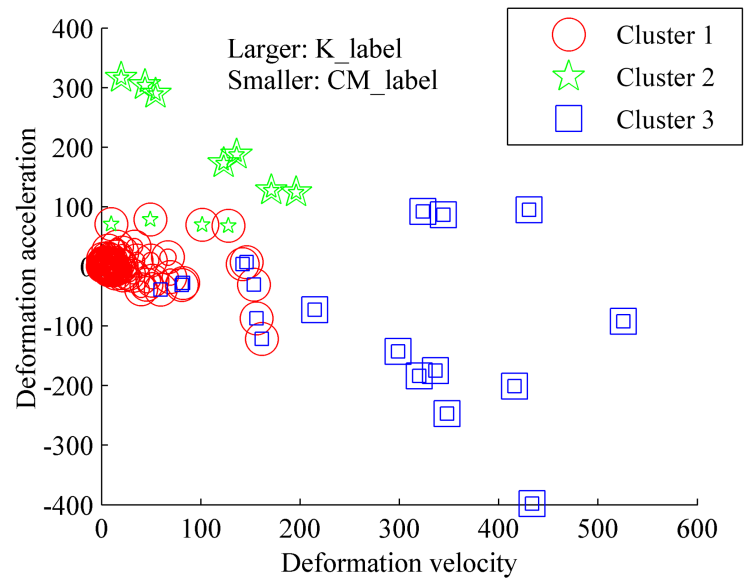

Figure 5. Comparison of K_label and CM_label.

high instability of a landslide and should draw immediate attention and early warning. Therefore, entropy analysis is conducted to analyse landslide instability based on deformation states. Focussing the timeliness and Markov property of deformation state sequence, state occurrence entropy (SOE) and state transition entropy (STE) are defined. Thereof, the product of SOE, STE and membership is defined as the SFE to describe the comprehensive information about landslide instability.

SOE mainly aims to measure the information about landslide stability provided by a single occurrence of one deformation state. Considering the great significance of severe deformation to landslide early warning, the basic equation of information entropy is modified to emphasise the probability difference between severe and slight deformation. To show the deformation tendency, the sign of SOE is defined to be the same as DT, which also reflects the timeliness of deformation states. State occurrence entropy is defined in Eq. (4).

$\mathrm{SOE}_{i, t}=\frac{-\log \left(p_{i}\right) / N_{i}}{\sum_{i=1}^{K}-\log \left(p_{i}\right) / N_{i}} \cdot \operatorname{sign}\left(\mathrm{DT}_{t}\right)$,

where $p_{i}$ is the probability of deformation state $i ; N_{i}$ is the frequency of deformation state $i ; K$ is the number of deformation states, i.e., the cluster number in the $K$-means clustering method; $\mathrm{DT}_{t}$ is the deformation tendency index (DT) at time $t$; and $\mathrm{SOE}_{i, t}$ is the state occurrence entropy of the occurrence of state $i$ at time $t$.

STE focuses on the measurement of the information about landslide stability when one deformation state transmits to another. Markov property describes such a property of a discrete state sequence as each state only being influenced by the former one state, independent of other states (Tauchen, 1986). Because the influence of external factors on landslide has the continuity and hysteresis characteristic, the deformation state sequence satisfies the Markov property. Therefore, the state transition matrix of the Markov chain is employed to quantitatively analyse the transition regularities of defor- 
Table 1. Monthly horizontal displacement of A3 from January 1978 to May 1985 (in millimetres).

\begin{tabular}{|c|c|c|c|c|c|c|c|c|c|c|c|c|}
\hline & Jan & Feb & Mar & Apr & May & Jun & Jul & Aug & Sep & Oct & Nov & Dec \\
\hline 1978 & 0.0 & 3.2 & 7.1 & 6.1 & 6.4 & 8.7 & 13.1 & 6.9 & 4.8 & 1.8 & 1.8 & 7.5 \\
\hline 1979 & 1.6 & 7.2 & 1.0 & 1.6 & 5.0 & 10.5 & 5.7 & 19.7 & 336.3 & 161.3 & 39.8 & 1.6 \\
\hline 1980 & 14.3 & 11.1 & 7.8 & 4.2 & 9.7 & 9.7 & 80.8 & 49.5 & 59.5 & 20.7 & 9.4 & 18.7 \\
\hline 1981 & 22.9 & 6.9 & 6.2 & 10.6 & 6.5 & 5.0 & 3.4 & 10.2 & 8.6 & 11.7 & 6.8 & 1.9 \\
\hline 1982 & 8.2 & 7.5 & 6.8 & 33.2 & 66.7 & 82.2 & 54.5 & 344.2 & 430.6 & 525.6 & 433.5 & 35.3 \\
\hline 1983 & 45.3 & 15.0 & 31.8 & 16.4 & 20.0 & 20.8 & 43.9 & 348.1 & 101.3 & 171.2 & 298.7 & 156.2 \\
\hline 1984 & 69.1 & 51.3 & 27.6 & 15.5 & 49.0 & 127.9 & 196.0 & 320.1 & 136.1 & 413.8 & 325.8 & 214.6 \\
\hline 1985 & 142.1 & 146.1 & 153.3 & 123.0 & 296.1 & & & & & & & \\
\hline
\end{tabular}

Table 2. Cluster centroids and number of items in each cluster.

\begin{tabular}{lrrr}
\hline K_label & Velocity $(v)$ & Acceleration $(a)$ & Items \\
\hline 1 & 30.83 & 0.13 & 69 \\
2 & 133.53 & 201.86 & 8 \\
3 & 366.75 & -133.09 & 10 \\
\hline
\end{tabular}

mation states. STE is defined as Eq. (5).

$$
\mathrm{STE}_{i j}=\frac{-p_{i j} \cdot \log \left(p_{i j}\right)}{\sum_{j=1}^{K}-p_{i j} \cdot \log \left(p_{i j}\right)}
$$

where $p_{i j}$ is the transition probability from former state $i$ to current state $j ; K$ is the number of deformation states, i.e., the cluster number in $K$-means clustering method; and $\mathrm{STE}_{i j}$ is the state transition entropy of the transition from former state $i$ to current state $j$. As for landslide deformation states, severe deformation occurs rarely, resulting in a small probability of transitions from other deformation states to severe deformation. In contrast, severe deformation indicates a high instability of a landslide and thus has a characteristic of poor sustainability. Apparently, the longer the severe deformation lasts, the higher instability the landslide and the larger STE will have.

Finally, SFE is defined as the product of state occurrence entropy, state transition entropy and membership degree, as shown in Eq. (6). This definition is mainly based on the following reasons: (1) although state occurrence entropy and state transition entropy emphasise the different attributes of deformation states, they are both expressed in the form of information entropy. (2) They share the common engineering significance that the larger the entropy, the more instable the landslide. (3) The membership in the cloud model indicates the extent to which displacement data support the deformation state concept and thus deserve consideration. Essentially, SFE is the individual contribution of temporary deformation state to a landslide's overall instability. By accumulating SFE according to time, cumulative state fusion entropy
(CSFE) can be obtained.

$$
\begin{aligned}
& \mathrm{SFE}_{j, t}=\mathrm{SOE}_{j, t} \cdot \mathrm{STE}_{i j} \cdot \mathrm{MBS}_{j} \\
& \mathrm{CSFE}_{t}=\sum_{t_{0}}^{t} \mathrm{SFE}_{j, t}
\end{aligned}
$$

\subsection{Result interpretation of state fusion entropy}

SFE is the comprehensive representation of the timeliness, Markov property and fuzziness attributes of deformation states. In mathematical form, SFE can be regarded as the weighted information amount, indicating the individuation of each deformation state to overall landslide instability. For the value, on the one hand, the sigh of SFE is determined by DT, indicating the deformation tendency of landslide. Positive DT indicates a growing instability, and negative DT indicates a decreasing instability. On the other hand, the instable extent is represented by the absolute value of SFE.

CSFE is the sum of SFE, as shown in Eq. (6). According to information theory, entropy indicates the overall uncertainty and instability of source. Likewise, CSFE reflects the overall instability of landslide in the whole monitoring period. In other words, CSFE represents the cumulative effect of landslide instability. As time goes on, CSFE will also indicate the changing regularities of landslide instability. If landslide stays in a slight deformation period, CSFE will remain at a relatively low level. If landslide develops into a severe deformation period, CSFE will accordingly show a continuous growth. Besides, a historical maximum index is introduced to identify key time nodes of stability changes. It is defined as the maximum from the very beginning to the time in question of CSFE. Each renewal of historical maximum suggests a more dangerous state of landslide. Once the new historical maximum occurs frequently, the CSFE curve will inevitably increase significantly, indicating high instability of a landslide. In this case, field surveys will be necessary for landslide early warning and hazard prevention.

\section{Case study}

To verify the effectiveness of the SFE method, five landslides in the Three Gorges Reservoir area in China were selected as 
Table 3. State transition entropy of Xintan landslide.

\begin{tabular}{lrrr}
\hline Deformation state & S1 & S2 & S3 \\
\hline S1 & 0.2679 & 0.4687 & 0.2634 \\
S2 & 0.0000 & 0.5516 & 0.4484 \\
S3 & 0.3635 & 0.3112 & 0.3253 \\
\hline
\end{tabular}

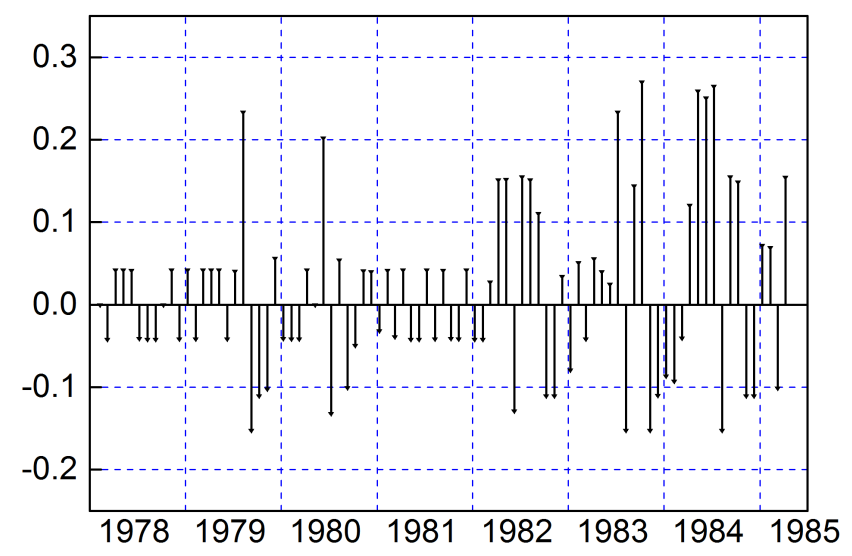

Figure 6. Monthly state fusion entropy of Xintan landslide.

examples for stability changing regularities analysis. Among them, the Xintan landslide is a reactive landslide triggered by rainfall. The Baishuihe landslide, Bazimen landslide and Shuping landslide are reactive landslides mainly triggered by reservoir water level and rainfall. Pajiayan landslide is a newly created landslide. Limited by space, the results of the Xintan landslide are detailed while others are simply presented.

The Xintan landslide, which occurred $26.6 \mathrm{~km}$ upstream of the Three Gorges Dam and $15.5 \mathrm{~km}$ downstream of Zigui County, is located in the city of Xintan on the north shore of Yangtze River. It extends from south to north with a length of $2000 \mathrm{~m}$. The width of the rear edge is about $300 \mathrm{~m}$ and the width of the front edge is between 500 and $1000 \mathrm{~m}$, with an average width of $450 \mathrm{~m}$. The elevation decreases from about $900 \mathrm{~m}$ in the north to $65 \mathrm{~m}$ in the south with an average gradient of about $23^{\circ}$. The main body of the deep-seated landslide was comprised of colluvial deposits overlying the bedrock of shale stone of Silurian system, sandstone of Devonian system and limestone of Carboniferous and Permian systems. The strike of the bedrock strata was mainly $10^{\circ} \mathrm{N}-30^{\circ} \mathrm{E}$, almost perpendicular to the Yangtze River. At the end of 1977, a monitoring system of surface displacement composed of four collimation lines was set up and eight markers were added in July 1984 mainly by the Avalanche Survey Department of Xiling Gorge. Thanks to this monitoring and field investigation, the losses were contained at the possible minima, without any fatalities and injuries when Xintan landslide occurred on 12 June 1985 (Zhang et al., 2006; Huang et al., 2009; Lin et al., 2013). According to previous studies, cumu-

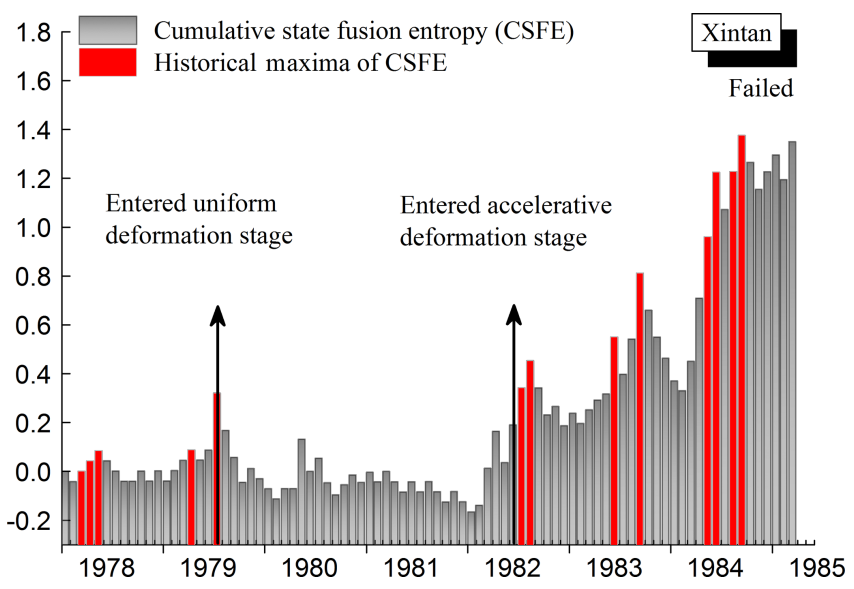

Figure 7. Cumulative state fusion entropy and historical maxima of Xintan landslide.

lative horizontal displacements at A3 and B3 are considered to be the most representative (Wang, 2009). Location and two monitoring points of Xintan landslide are shown in Fig. 3. According to previous studies (He et al., 2015), monthly horizontal displacement of A3 from January 1978 to May 1985 is shown in Table 1. Since the displacement of A3 was obtained monthly, deformation states and state fusion entropy will also be monthly indexes for Xintan landslide.

Considering that the monitoring error of GPS can be ignored compared to landslide actual deformation on a monthly timescale, monthly deformation velocity $(v)$ was selected as the DE index and monthly deformation acceleration $(a)$ as the DT index. Firstly, monthly deformation states were defined based on a joint clustering method of $K$-means and a cloud model, with monthly deformation velocity and acceleration as the inputs. Given that there are about 90 monthly items with two dimensions, i.e., $(v, a)$, cluster number $K$ was empirically set to 3 for simplicity. The initial cluster centroids were determined by performing preliminary clustering phase on a random $10 \%$ subsample of data set. The clustering process was repeated nine times and the cluster labels (K_label) were determined based on voting strategy. Cluster centroids and number of items in each cluster are shown in Table 2.

As can be seen from Table 2, obvious numerical differences exist among cluster centroids, suggesting different deformation patterns. Most items belong to the first cluster, whose deformation velocity and acceleration remain at a relative low level, proving that the occurrence probability of slight deformation is large while that of severe deformation is small during the development of landslide.

The cloud model continued to evaluate the membership of each item to the corresponding cluster label. In view of the non-negative numerical limit of deformation velocity, cluster 1 was set as a right half cloud, cluster 2 as a symmetric cloud and cluster 3 as a left half cloud in deformation velocity 


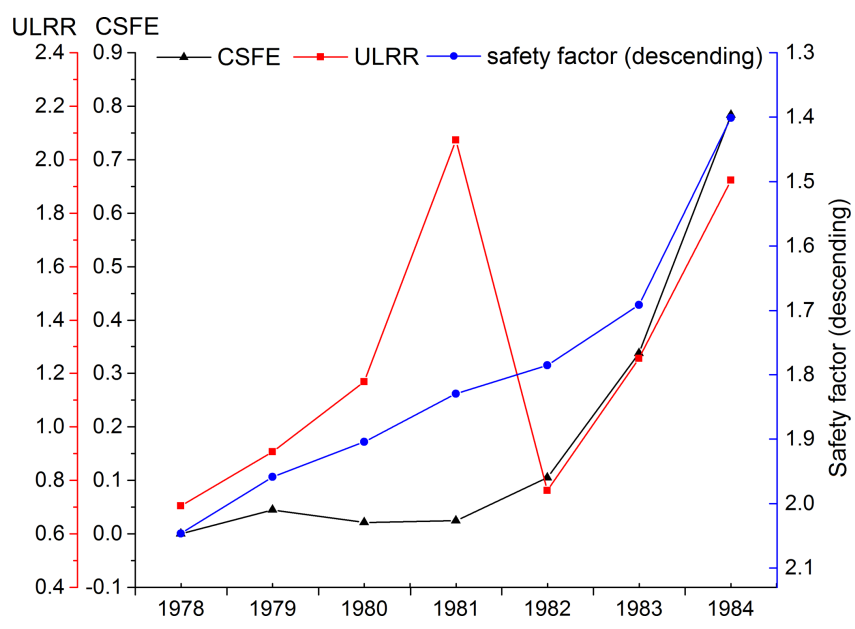

Figure 8. Comparison of CSFE with ULRR and safety factor of Xintan landslide.

dimension. In deformation acceleration dimension, all clusters were set as symmetric clouds. The regenerated clouds are shown in Fig. 4. After obtaining digital features of each cloud, membership of each item to all clouds was calculated and unified, and the cloud label (CM_label) with the largest membership was defined as the monthly deformation state. Comparison of K_label and CM_label is shown in Fig. 5. As can be seen, K_label and CM_label are almost the same. However, there are some items which belonged to cluster 1 in $K$-means that now belong to cluster 2 or 3 in the cloud model, indicating that cluster 1 has a low tolerance for numerical deviations.

After the joint clustering process of $K$-means and the cloud model, monthly deformation states were derived, after which SFE analysis of deformation state sequence was followed. As mentioned in the Methods section, state occurrence entropy and state transition entropy are defined with relation to the timeliness and Markov property of deformation states. After the statistics of the frequency and probability of each deformation state, state occurrence entropy of each deformation state was calculated based on Eq. (4), whose absolute values were $0.1621,0.4980$ and 0.3399 . A state transition matrix was obtained by analysing deformation state sequence and state transition entropy obtained based on Eq. (5) is shown in Table 3. Three values are mainly discussed here. (1) The STE from S2 to S1 is zero. As mentioned earlier, S2 has a relatively large deformation velocity while S1 has a smaller one. Thus, a deceleration process which corresponds to S3 will inevitably show up between S2 and S1. (2) The transition from slight deformation S1 to S1 presents a small transition entropy, indicating a small risk of landslide. (3) The maximum transition entropy occurs in the transition from $S 2$ to $S 2$, indicating an increasing instability.

Finally, monthly SFE was calculated based on Eq. (6), as shown in Fig. 6. Between December 1977 and December 1981, monthly SFE remains at a low level, fluctuating around zero. There are two local maxima but they only last a short time. Between January 1982 and May 1982, values close to the local maxima in earlier stage occur frequently, indicating increasing instability and higher risk of the Xintan landslide.

For an insight into the cumulative effect and changing regularities of landslide instability, CSFE was calculated, after which historical maxima were picked out, as shown in Fig. 7. As for the CSFE curve, there are two typical changing forms: fluctuation around zero type and fluctuant increasing type. The first type occurs between January 1978 and February 1982, during which the CSFE fluctuates around zero with a slight decrease. A local maximum occurs in $\mathrm{Au}-$ gust 1979. The global minimum occurs in February 1982. After February 1982, CSFE shows an apparent fluctuant increasing trend. Historical maxima mainly concentrate in two periods. From January 1978 to July 1979, the first period is at the prophase of the monitoring period and the historical maximum is relatively small and easy to update. From June 1982 to April 1985, the second period is at the anaphase of the monitoring period. During this time, the frequent renewal of the historical maximum indicates an increasing instability of Xintan landslide and higher risk of landslide hazard.

The macroscopic behaviours of Xintan landslide near historical maxima were investigated according to previous studies (Wang, 1996). Around August 1982, the front edge of Jiangjiapo went through a small collapse. In June 1983, the colluvial deposits between Guangjiaya and Jiangjiapo showed signs of resurrection. At the end of 1984, the trailing edge of the landslide showed an "armchair" shape and the leading edge was bulged out. Some collapse pits were found on the upper side while several new tensile cracks were found in the middle. Meanwhile, some small collapses which seem irrelevant to rainfall occurred. In May 1985, old cracks widened and new cracks appeared, forming a ladder-shaped landing ridge. Moreover, Jiangjiapo presented a clear trend of the overall slippage. These proofs suggest that the historical maximum index is highly consistent with landslide macroscopic deformation behaviours.

Many studies have claimed the close relationship between landslide stability and evolutionary stages (Xu et al., 2008). Thus the evolutionary stages of Xintan landslide were introduced to verify the effectiveness of the SFE method. According to previous studies, Xintan landslide entered uniform deformation stage in August 1979, entered accelerative deformation stage in July 1982 and failed in June 1985 (Yin et al., 2002). As shown in Fig. 7, August 1979 corresponds to a local mutation of CSFE and is also the end of the first period of historical maxima. July 1982 is located at the fluctuant increasing period of CSFE and it is the start of the second period of historical maxima. Before the failure of Xintan landslide, CSFE had already reached a really high level in April 1985. In other words, historical maxima match really well with the evolutionary stages of Xintan landslide in key time nodes and may suggest the effectiveness of this method. 

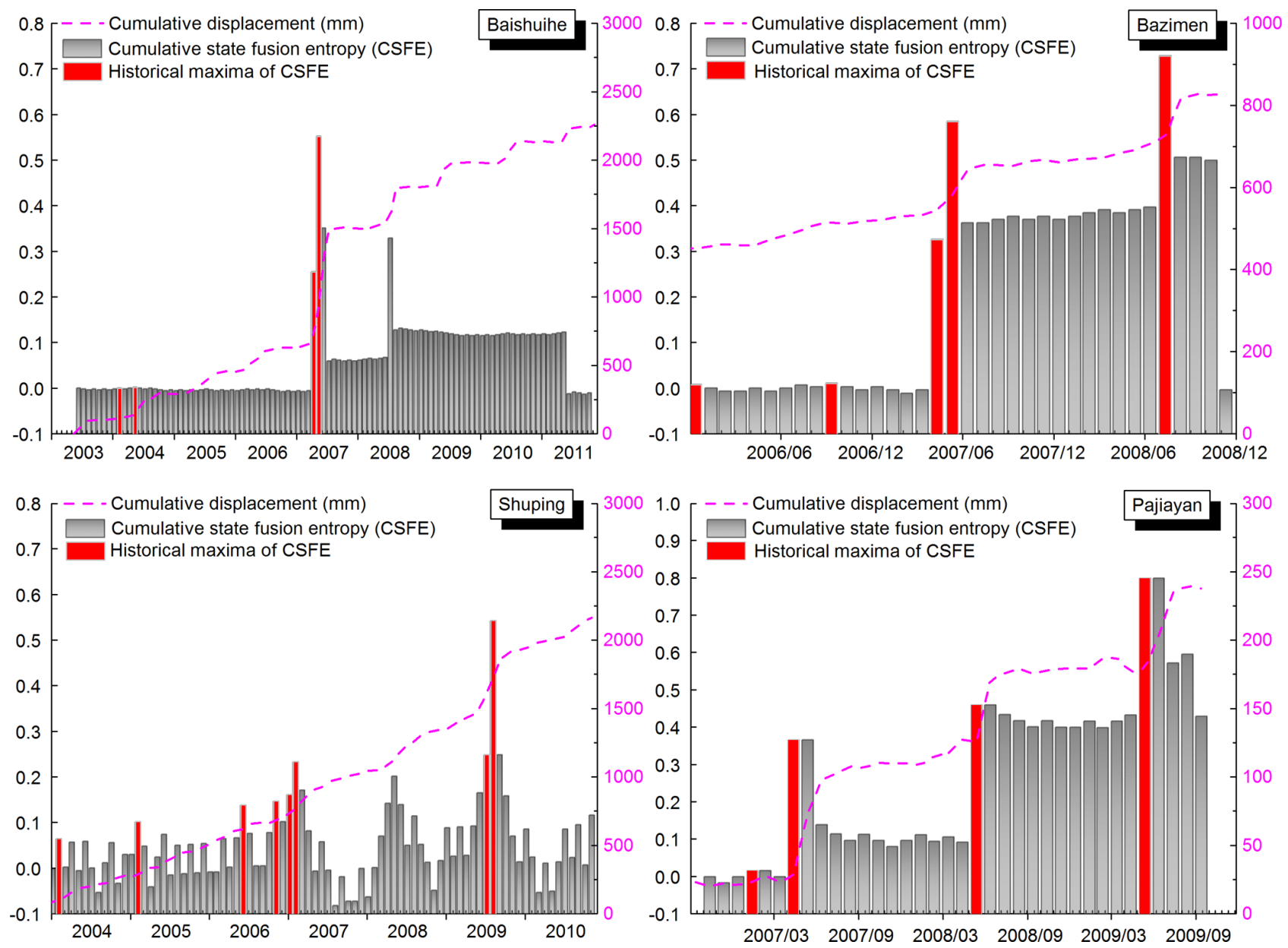

Figure 9. Cumulative state fusion entropy and historical maxima of Baishuihe, Bazimen, Shuping and Pajiayan landslides.

Furthermore, when Xintan landslide entered accelerative deformation stage in July 1982, CSFE started an obvious fluctuant increasing trend. In this aspect, the fluctuant increasing type of CSFE may serve as a new clue to determine whether a landslide is entering the accelerative deformation stage or not.

In addition, the results of several other studies were introduced for comparison. Chen (2014) studied the stability of Xintan landslide by FEM with consideration of the loading effect and material weakening caused by rainfall, as shown in Fig. 8. Moreover, the result of an unloading-loading response ratio method (ULRR) is also introduced (Zhang et al., 2006; He et al., 2010), as shown in Fig. 8. Because only annual results are given in these studies, annual averages of CSFE were correspondingly calculated for comparison. According to Fig. 8, the safety factor decreases year by year and cannot reflect the recovery process of landslides stability. The ULRR presents similar changing regularities like CSFE after Xintan landslide entered the accelerative deformation stage in 1982. However, the mutation in 1981 when Xintan is still in the uniform deformation stage seems unreasonable. Be- sides, ULRR is obtained yearly and offers less details about stability changes than CSFE.

Similarly, SFE analysis of Baishuihe landslide, Bazimen landslide, Shuping landslide and Pajiayan landslide in the Three Gorges Reservoir area in China was also conducted on the displacement data that derived from previous studies (Tang et al., 2012; Xu, 2012; Xu et al., 2013; Liao, 2014) and the results are shown in Fig. 9. Similarities and differences between displacement and state fusion entropy are found through a comparative analysis of these landslides. For the Bazimen landslide and Pajiayan landslide, CSFE and cumulative displacement show similar change rules, especially during the drawdown period of water level, indicating their intrinsic consistency. For the Baishuihe landslide and Shuping landslide, CSFE shows a distinctly different characteristic from their cumulative displacement. Taking Baishuihe landslide as an example, the severe deformation in June 2007 seems to suggest that the landslide entered the accelerative deformation stage. However, subsequent monitoring has proven that the deformation is only a temporary effect of heavy rainfall and fluctuation of water level (Xu et al., 2008). 

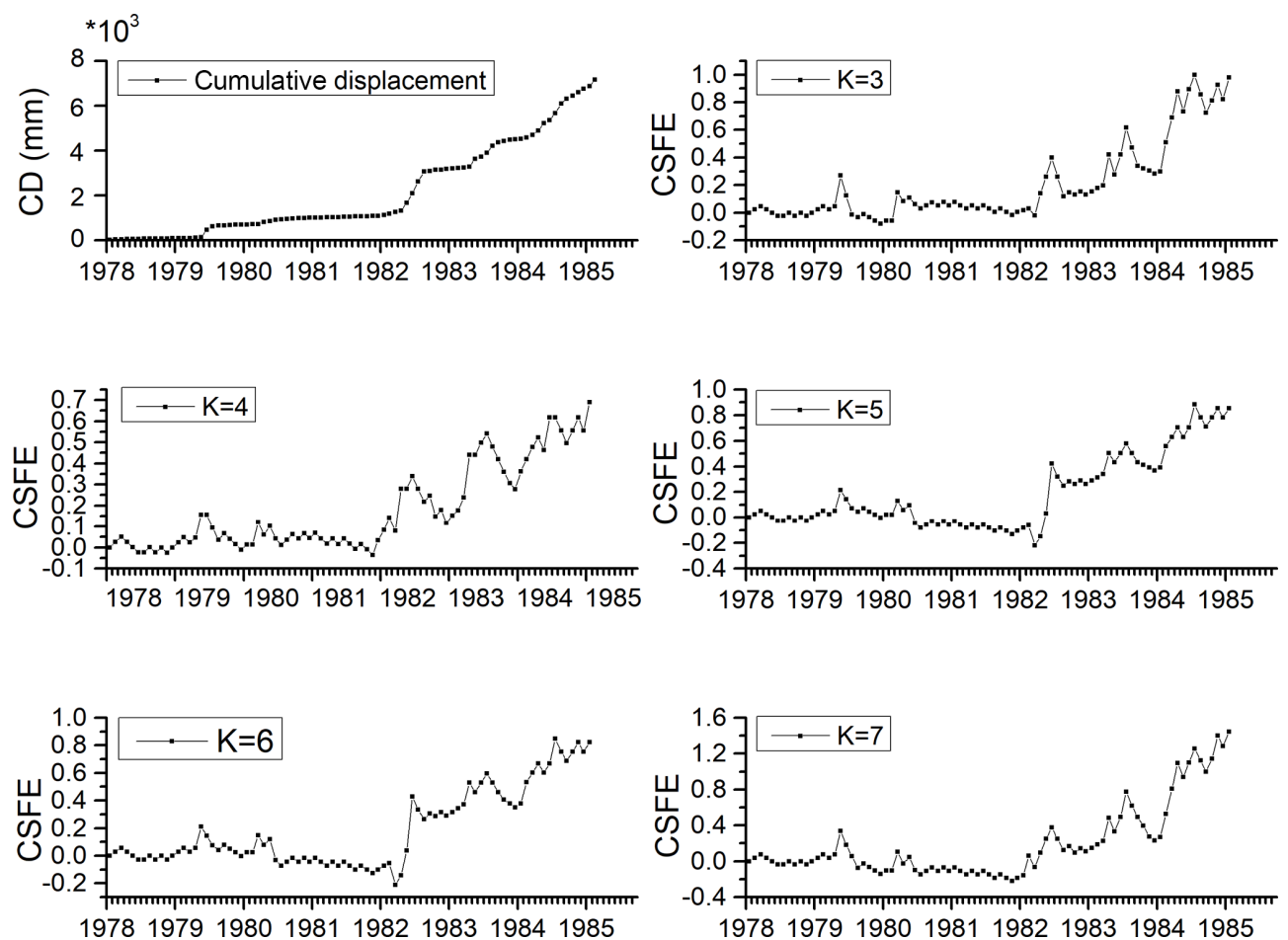

Figure 10. CSFE with different cluster numbers ( $K=3$ to 7$)$ of Xintan landslide.

In Fig. 9, CSFE of Baishuihe landslide returns to a low level after several historical maxima.

\section{Discussion and conclusion}

Under the guidance of dynamic state system and based on the relationship of displacement monitoring data, deformation state and landslide stability, a state fusion entropy approach is proposed to conduct a continuous and site-specific analysis of the changing regularities of landslide stability. A joint clustering method combining $K$-means and a cloud model is firstly proposed to investigate landslide deformation states, and then a multi-attribute entropy analysis follows to estimate landslide instability. Furthermore, a historical maximum index is introduced for identifying key time nodes of stability changes. To verify the effectiveness of this approach, Xintan landslide is selected as a detailed case and four other landslides in the Three Gorges Reservoir area as brief cases. Taking Xintan landslide as an example, cumulative state fusion entropy mainly fluctuated around zero in the initial deformation stage and uniform deformation stage, but an obvious fluctuant increasing tendency appeared after Xintan landslide entered accelerative deformation stage. Additionally, a thorough collection of the macroscopic proofs also suggests that historical maxima are highly consistent with landslide macroscopic deformation behaviours.

Compared with traditional safety factor, state fusion entropy evaluates the landslide instability and is capable of in- dicating its extent and changing regularities. Compared with simulation methods for landslide stability analysis, this approach takes displacement monitoring data as the basis of landslide stability analysis and thus is prone to continuous stability analysis. Compared with direct judgment from displacement monitoring data, this approach analyses landslide deformation states by using a data-driven model, avoiding the disunity of individual engineering geology experience, ensuring its applicability to the geological conditions of different landslides.

To measure the influence of different cluster numbers on the performance of this method, CSFE with different cluster numbers ( $K=3$ to 7 ) is compared, as shown in Fig. 10. As can be seen, CSFE varies slightly with $K$. This is mainly because different $K$ correspond to different division roughness of deformation states, which sequentially affects the value of the CSFE. Due to this fluctuation, CSFE is not intended to be applied to landslide early warning unless it is qualified by further research. Despite this, the overall trend remains unchanged, which suggests a steady statistical regularity of deformation states.

Furthermore, several issues also need to be clarified. Firstly, data selection and feature extraction are simplified. Multiple monitoring has already become a common practice in landslide monitoring, but the comprehensive mining of multi-source data is also still a common problem and relevant research of SFE is still in progress. Despite this, some theories have already emerged. For the open system of land- 
slide, the displacement of different monitoring points can be regarded as landslide samples with different deformation scales. In contrast, fractal theory tells that same patterns as the entire system can be found if a small part of the whole is magnified. Therefore, fractal theory may contribute to multipoint data analysis. Due to the lack of higher time resolution monitoring data, a common practice of selecting one typical monthly displacement data of GPS has been adopted for now. On this timescale, deformation velocity and acceleration are considered to express landslide deformation well and are thus selected for deformation state definition. For higher time resolution data, some feature extraction methods may be necessary to determine the DE and DT indexes. Finally, entering into accelerative deformation stage is a necessary condition for landslide failure. The fluctuant increasing tendency of cumulative state fusion entropy and the frequent renewal of historical maximum may help to judge whether a landslide has entered accelerative deformation stage. Once this happens, other clues such as macro-cracks should also be taken into account to fully determine landslide early warning level.

Data availability. The research data used in the paper are mainly derived from several published Chinese papers, as well as the monthly monitoring reports written by our team. For each of the following landslides, a source is available: Xintan landslide (He et al., 2015), Baishuihe landslide (Liao, 2014), Bazimen landslide (Tang et al., 2012), Shuping landslide (Xu et al., 2013), and Pajiayan landslide (Xu, 2012).

Competing interests. The authors declare that they have no conflict of interest.

Special issue statement. This article is part of the special issue "Landslide early warning systems: monitoring systems, rainfall thresholds, warning models, performance evaluation and risk perception". It is not associated with a conference.

Acknowledgements. This research was funded by the National Natural Sciences Foundation of China (grant numbers 41772376 and 41302278). The authors are grateful to the editors and reviewers for kind and constructive suggestions.

Edited by: Stefano Luigi Gariano

Reviewed by: Gianvito Scaringi and three anonymous referees

\section{References}

Ashland, F. X., Giraud, R. E., and McDonald, G. N.: Slope-stability implications of ground-water-level fluctuations in wasatch front landslides and adjacent slopes, Northern Utah, in: 40th Sym- posium on Engineering Geology and Geotechnical Engineering 2006, 24-26 May 2006, Logan, UT, USA, 33-44, 2006.

Bernardie, S., Desramaut, N., Malet, J. P., Gourlay, M., and Grandjean, G.: Prediction of changes in landslide rates induced by rainfall, Landslides, 12, 481-494, https://doi.org/10.1007/s10346014-0495-8, 2015.

Bordoni, M., Meisina, C., Valentino, R., Lu, N., Bittelli, M., and Chersich, S.: Hydrological factors affecting rainfallinduced shallow landslides: from the field monitoring to a simplified slope stability analysis, Eng. Geol., 193, 19-37, https://doi.org/10.1016/j.enggeo.2015.04.006, 2015.

Chen, G. Q., Huang, R. Q., Shi, Y. C., and Xu, Q.: Stability analysis of slope based on dynamic and whole strength reduction methods, Chinese Journal of Rock Mechanics and Engineering, 33, 243-256, https://doi.org/10.13722/j.cnki.jrme.2014.02.002, 2014.

Chen, H. C.: Study on dynamic load laws and attenuation laws of parameters induced by rainfall of colluvial landslide and its stability evolutional laws, Master thesis, Qingdao Technological University, 2014.

Dai, F. C., Lee, C. F., and Ngai, Y. Y.: Landslide risk assessment and management: an overview, Eng. Geol., 64, 65-87, https://doi.org/10.1016/S0013-7952(01)00093-X, 2002.

Dawson, E. M., Roth, W. H., and Drescher, A.: Slope stability analysis by strength reduction, Géotechnique, 49, 835-840, https://doi.org/10.1680/geot.1999.49.6.835, 2015.

Devkota, K. C., Regmi, A. D., Pourghasemi, H. R., Yoshida, K. Pradhan, B., Ryu, I. C., Dhital, M. R., and Althuwaynee, O. F.: Landslide susceptibility mapping using certainty factor, index of entropy and logistic regression models in GIS and their comparison at Mugling-Narayanghat road section in Nepal Himalaya, Nat. Hazards, 65, 135-165, https://doi.org/10.1007/s11069-0120347-6, 2013.

Duncan, J. M.: State of the art: limit equilibrium and finite-element analysis of slopes, J. Geotech. Eng.-ASCE, 122, 577-596, https://doi.org/10.1061/(ASCE)0733-9410(1996)122:7(577), 1996.

Federico, A., Popescu, M., Elia, G., Fidelibus, C., Internò, G., and Murianni, A.: Prediction of time to slope failure: a general framework, Environ. Earth Sci., 66, 245-256, https://doi.org/10.1007/s12665-011-1231-5, 2012.

Griffiths, D. V. and Fenton, G. A.: Probabilistic slope stability analysis by finite elements, J. Geotech. Geoenviron., 130, 507-518, https://doi.org/10.1061/(ASCE)1090-0241(2004)130:5(507), 2004.

Hartigan, J. A. and Wong, M. A.: A K-means clustering algorithm, Appl. Stat.-J. Roy. St. C, 28, 100-108, https://doi.org/10.2307/2346830, 2013.

He, K., Wang, S., Du, W., and Wang, S.: Dynamic features and effects of rainfall on landslides in the Three Gorges Reservoir region, China: using the Xintan landslide and the large Huangya landslide as the examples, Environ. Earth Sci., 59, 1267-1274, https://doi.org/10.1007/s12665-009-0114-5, 2010.

He, K. Q., Yang, D. B., Guo, L., and Li, J.: Prediction parameter of water dynamics coupled with displacement and evaluation method of debris landslide, Rock and Soil Mechanics, 36, 37-46, https://doi.org/10.16285/j.rsm.2015.S2.005, 2015.

Hsu, C. F. and Chien, L. K.: Slope stability analysis of transient seepage under extreme climates: case study of ty- 
phoon nari in 2001, J. Mar. Sci. Tech.-Taiw., 24, 399-412, https://doi.org/10.6119/jmst-015-0813-1, 2016.

Huang, F. M., Huang, J. S., Jiang, S. H., and Zhou, C. B.: Landslide displacement prediction based on multivariate chaotic model and extreme learning machine, Eng. Geol., 218, 173-186, https://doi.org/10.1016/j.enggeo.2017.01.016, 2017a.

Huang, F. M., Luo, X. Y., and Liu, W. P.: Stability analysis of hydrodynamic pressure landslides with different permeability coefficients affected by reservoir water level fluctuations and rainstorms, Water (Switzerland), 9, 1-16, https://doi.org/10.3390/w9070450, 2017b.

Huang, Z. Q., Law, K. T., Liu, H. D., and Jiang, T.: The chaotic characteristics of landslide evolution: a case study of Xintan landslide, Environ. Geol., 56, 1585-1591, https://doi.org/10.1007/s00254-008-1256-6, 2009.

Knappett, J. A.: Numerical analysis of slope stability influenced by varying water conditions in the reservoir area of the Three Gorges, China, Tenth International Symposium on Landslides and Engineered Slopes, 2008.

Li, D. Y. and Liu, C. Y.: Study on the universality of the normal cloud model, Engineering Science, 3, 28-34, 2004.

Li, D. Y., Meng, H. J., and Shi, X. M.: Membership clouds and membership clouds generators, Journal of Computer Research and Development, 32, 15-20, 1995.

Li, S. H., Liu, T. P., and Liu, X. Y.: Analysis method for landslide stability, Chinese Journal of Rock Mechanics and Engineering, 28, 3309-3324, 2009.

Liao, Y. Q.: Research on 3D Visual Simulation of the Landslide Evolution Based on Displacement, Master, Systems Engineering, Huazhong University of Science and Technology, available at: http://d.old.wanfangdata.com.cn/Thesis/D611178 (last access: 18 April 2018), 2014

Lin, D. C., Cai, J. L., Guo, Z. L., Zeng, F. L., An, F. P., and Liu, H. B.: Evaluation of landslide risk based on synchronization of nonlinear motions in observed data, Nat. Hazards, 65, 581603, https://doi.org/10.1007/s11069-012-0385-0, 2013.

Liu, Y., Liu, D., Qin, Z. M., Liu, F. B., and Liu, L.: Rainfall data feature extraction and its verification in displacement prediction of Baishuihe landslide in China, B. Eng. GeolEnviron., 75, 897907, https://doi.org/10.1007/s10064-015-0847-1, 2016.

Macciotta, R., Hendry, M., and Martin, C. D.: Developing an early warning system for a very slow landslide based on displacement monitoring, Nat. Hazards, 81, 887-907, https://doi.org/10.1007/s11069-015-2110-2, 2016.

Manconi, A. and Giordan, D.: Landslide early warning based on failure forecast models: the example of the Mt. de La Saxe rockslide, northern Italy, Nat. Hazards Earth Syst. Sci., 15, 16391644, https://doi.org/10.5194/nhess-15-1639-2015, 2015.

Montesarchio, V., Ridolfi, E., Russo, F., and Napolitano, F.: Rainfall threshold definition using an entropy decision approach and radar data, Nat. Hazards Earth Syst. Sci., 11, 2061-2074, https://doi.org/10.5194/nhess-11-2061-2011, 2011.

Montrasio, L., Valentino, R., and Losi, G. L.: Towards a real-time susceptibility assessment of rainfall-induced shallow landslides on a regional scale, Nat. Hazards Earth Syst. Sci., 11, 1927-1947, https://doi.org/10.5194/nhess-11-1927-2011, 2011.

Morales-Esteban, A., de Justo, J. L., Reyes, J., Azañón, J. M., Durand, P., and Martínez-Álvarez, F.: Stability analysis of a slope subject to real accelerograms by finite elements. Application to
San Pedro cliff at the Alhambra in Granada, Soil Dyn. Earthq. Eng., 69, 28-45, https://doi.org/10.1016/j.soildyn.2014.10.023, 2015.

Pourghasemi, H. R., Mohammady, M., and Pradhan, B.: Landslide susceptibility mapping using index of entropy and conditional probability models in GIS: Safarood Basin, Iran, Catena, 97, 7184, https://doi.org/10.1016/j.catena.2012.05.005, 2012.

Priest, G. R., Schulz, W. H., Ellis, W. L., Allan, J. A., Niem, A. R., and Niem, W. A.: Landslide stability: role of rainfall-induced, laterally propagating, porepressure waves, Environ. Eng. Geosci., 17, 315-335, https://doi.org/10.2113/gseegeosci.17.4.315, 2011.

Ridolfi, E., Montesarchio, V., Russo, F., and Napolitano, F.: An entropy approach for evaluating the maximum information content achievable by an urban rainfall network, Nat. Hazards Earth Syst. Sci., 11, 2075-2083, https://doi.org/10.5194/nhess11-2075-2011, 2011.

Saito, M.: Forecasting the time of occurrence of a slope failure, in: Proceedings of 6th International Congress of Soil Mechanics and Foundation Engineering, Montreal, 537-541, 1965.

Shannon, C. E.: A mathematical theory of communication, Bell Syst. Tech. J., 27, 379-423, https://doi.org/10.1002/j.15387305.1948.tb01338.x, 1948.

Shi, Y. F. and Jin, F. X.: Landslide stability analysis based on generalized information entropy, in: International Conference on Environmental Science and Information Application Technology, Wuhan, China, 83-85, 2009.

Singh, A. K., Kainthola, A., and Singh, T. N.: Prediction of factor of safety of a slope with an advanced friction model, International Journal of Rock Mechanics \& Mining Sciences, 55, 164-167, https://doi.org/10.1016/j.ijrmms.2012.07.009, 2012.

Steinley, D.: K-means clustering: a half-century synthesis, Brit. J. Math. Stat. Psy., 59, 1-34, https://doi.org/10.1348/000711005X48266, 2006.

Tang, L. S., Yin, K. L., and Du, J.: Time Prediction for Landslides with Step-Style Displacement Characteristics, Geological Science and Technology Information, 31, 106-110, available at: http://en.cnki.com.cn/Article_en/CJFDTotal-DZKQ201204019. htm (last access: 18 April 2018), 2012.

Tauchen, G.: Finite state markov-chain approximations to univariate and vector autoregressions, Econ. Lett., 20, 177-181, https://doi.org/10.1016/0165-1765(86)90168-0, 1986.

van Asch, Th. W. J., Malet, J.-P., and Bogaard, T. A.: The effect of groundwater fluctuations on the velocity pattern of slowmoving landslides, Nat. Hazards Earth Syst. Sci., 9, 739-749, https://doi.org/10.5194/nhess-9-739-2009, 2009.

Wang, N. Q., Xue, Y. Q., Yu, Z., and Feng, X.: Review of landslide stability analysis method, Adv. Mat. Res., 1004-1005, 15411546, https://doi.org/10.4028/www.scientific.net/AM R.10041005.1541, 2014.

Wang, S. Q.: Review on prediction of Xintan landslide, The Chinese Journal of Geological Hazard and Control, 5, 1119, https://doi.org/10.16031/j.cnki.issn.1003-8035.1996.s1.003, 1996.

Wang, S. Q.: Time prediction of the Xintan landslide in Xiling Gorge, the Yangtze River, in: Landslide Disaster Mitigation in Three Gorges Reservoir, China, edited by: Wang, F. W. and Li, T. L., Springer, Berlin, Heidelberg, 411-431, 2009. 
Wu, X. L., Zhan, F. B., Zhang, K. X., and Deng, Q. L.: Application of a two-step cluster analysis and the Apriori algorithm to classify the deformation states of two typical colluvial landslides in the Three Gorges, China, Environ. Earth Sci., 75, 146, https://doi.org/10.1007/s12665-015-5022-2, 2016.

$\mathrm{Xu}, \mathrm{F}$. : Research of Induced Mechanism and Prediction of Pajiayan Landslide in Zhong County in Three Gorges Reservoir Area, Master, Geological Engineering, China University of Geosciences, available at: http://d.old.wanfangdata.com.cn/Thesis/ Y2190169 (last access: 18 April 2018), 2012.

$\mathrm{Xu}, \mathrm{Q}$. and Zeng, Y. P.: Research on acceleration variation characteristics of creep landslide and early-warning prediction indicator of critical sliding, Chinese Journal of Rock Mechanics and Engineering, 28, 1099-1106, 2009.

Xu, Q., Tang, M. G., Xu, K. X., and Huang, X. B.: Research on space-time evolution laws and early warning-prediction of landslides, Chinese Journal of Rock Mechanics and Engineering, 27, 1104-1112, 2008.
Xu, X. X., Niu, R. Q., Ye, R. Q., and Wang, J. W.: Displacement Prediction Model of Landslide Based on Trigger Factors Analysis, Journal of Yangtze River Scientific Research Institute, 30, 42-47, https://doi.org/10.3969/j.issn.1001-5485.2013.07.009, 2013.

Yin, K. L., Jiang, Q. H., and Wang, Y.: Numerical simulation on the movement process of Xintan landslide by DDA method, Chinese Journal of Rock Mechanics and Engineering, 21, 959-962, 2002.

Zhang, W. J., Chen, Y. M., and Zhan, L. T.: Loading/unloading response ratio theory applied in predicting deep-seated landslides triggering, Eng. Geol., 82, 234-240, https://doi.org/10.1016/j.enggeo.2005.11.005, 2006. 